\title{
Review Article \\ On the Issues and Challenges of Fiber-Wireless (Fi-Wi) Networks
}

\author{
Harry Tin Win and Al-Sakib Khan Pathan \\ Department of Computer Science, International Islamic University Malaysia (IIUM), Jalan Gombak, 53100 Kuala Lumpur, Malaysia
}

Correspondence should be addressed to Al-Sakib Khan Pathan; sakib.pathan@gmail.com

Received 16 December 2012; Revised 16 March 2013; Accepted 27 March 2013

Academic Editor: Qishi Wu

Copyright (C) 2013 H. T. Win and A.-S. K. Pathan. This is an open access article distributed under the Creative Commons Attribution License, which permits unrestricted use, distribution, and reproduction in any medium, provided the original work is properly cited.

\begin{abstract}
The objective of this article is to present various aspects of Fiber-Wireless (Fi-Wi) networks, which is a technology of current interest of the researchers. Fiber-wireless technology, known in short as "Fi-Wi", is a combination of optical-fiber-based network and wireless network. Fi-Wi has recently come forward as one of the emerging future network technologies yet the term is often mixed up with wireless-fidelity (Wi-Fi) technology. In fact, Wi-Fi network could be included in a Fi-Wi architecture. Due to the unfamiliarity with the term, "Fi-Wi" and similar sounding commonly known "Wi-Fi", many students, even academics and practitioners sometimes may not be able to differentiate between these. In the recent years, a good number of works on various issues of Fi-Wi have come out, but an overall survey with the latest advancements in the area is yet to be found. Hence, in this work, we analyze various issues and challenges of implementing Fi-Wi networks in practical scenarios to allow researchers to find future directions of research and general readers to understand the issues better.
\end{abstract}

\section{Introduction}

Fiber-wireless (Fi-Wi) or optical wireless is the combined use of "optical" (optical fiber) and "wireless" (radio frequency) communication to provide telecommunication services to the clusters of end points which are geographically distant. It is the combination of passive optical networks (PONs) and wireless mesh networks (WMNs). Optical networks are designed to provide long-distance, high-bandwidth communications with the help of wireless networks to provide ubiquitous, flexible communications in community areas. The high-capacity optical fiber is used to span the longest distances, and a lower cost wireless link carries the signal for the last mile to nearby users. Fi-Wi network can support various types of communications such as upstream, downstream, and peer-to-peer (P2P) communication.

A Fi-Wi network consists of a wireless subnetwork as the front end and an optical subnetwork as the back end. A WMN consists of multiple gateways to the Internet access and a group of wireless mesh routers with multiplewireless mesh clients. PON subnetwork in Fi-Wi network can provide up to 1.25 Gbps EPON (Ethernet PON) and GPON (Gigabit PON) supporting $2.488 \mathrm{Gbps}$ rate in the downstream and $1.244 \mathrm{Gbps}$ rate in the upstream which are very high bandwidth facilities, but the network still faces the impact of interferences in wireless communications which often lead to severely limiting the network throughput in wireless subnetworks. The effect of interference on network throughput can be reduced by the integrated feature of peer-to-peer communication from one wireless client to another wireless client in Fi-Wi network, which has become a focus point in IEEE $802.11 \mathrm{~s}$ standard for WMNs [1]. When peer-to-peer communication is used in Fi-Wi network, network throughput can be increased by increasing traffic for each flow that each P2P communication flow could generate more demand of network packets to increase the overall throughput. By using this method, the delay in P2P communication can be reduced since the transmission in the PON subnetwork can be much faster than that is in the multihop wireless network part. We will discuss these issues later in this paper. 
1.1. Objectives of This Paper. A major objective of this work is to analyze the architecture of Fi-Wi network and its various aspects. The integration of different technologies can be addressed for low cost of deployment, high bandwidth provision, and scalable extension of communication coverage. Such wireless-optical-wireless communication mode introduced by Fi-Wi network can sustain the interference in wireless subnetwork because packets of flow are sent through multiple paths in wireless network and then, these packets are sent to optical line terminal (OLT) through different optical network units (ONUs) in the optical subnetwork. Another objective of the work is to understand the observations for network throughput gain of Fi-Wi network in P2P communication and the traffic to the Internet. Our purpose is to explore Fi-Wi network from various angles, talk about the critical performance metrics, and make it easy to grasp by the research community and general readers. Fi-Wi could be the nextwave of speedy wireless communication [2], yet, in reality, there are a very few survey works on this topic $[3,4]$, and with given the current advancements and trends, we deem it necessary to address various issues with latest achievements in this area.

1.2. Structure of This Paper. This paper is organized as follows. After Section 1, Section 2 talks about the structural aspects of Fi-Wi network. Section 3 presents the issues that were investigated in various available literatures, Section 4 talks about Quality of Service (QoS) issues in Fi-Wi network while Section 5 talks about transmission control protocol (TCP) performance issues. Section 6 discusses traffic routing issues. These are the major issues for which Fi-Wi is thought to replace the relatively slow today's networks. Section 7 is our overall finding of the areas of potential research and challenge. Section 8 concludes the paper.

\section{Structural Aspects of Fi-Wi Network}

To understand and analyze the Fi-Wi network architecture, the concepts of passive optical network, underlying technologies, and traditional wireless network are needed to be understood. Before proceeding further, in Table 1, we put the major terminologies used in this paper alongside their meanings for the ease of reading.

2.1. Network Architecture for Fi-Wi Based P2P Communication. In a Fi-Wi network, passive optical network consists of an optical line termination (OLT) at the communication company's office and a number of optical network units (ONUs) near end users. Typically, up to 32 ONUs can be connected to an OLT through Remote Note (RN). An ONU is connected to the mesh clients through wireless mesh routers. A peer-to-peer ( $\mathrm{P} 2 \mathrm{P})$ communication in such network can be carried through the wireless-optical-wireless mode in which the traffic is sent from the source wireless client to its nearest ONU, which is then sent to the closest ONU of the destination wireless client through the PON subnetwork, and then traffic is delivered to the destination wireless client. For the traffic route to the Internet, the traffic is sent from mesh client to
TABLE 1: Major terminologies at a glance.

\begin{tabular}{ll}
\hline Abbreviations & Full meaning \\
\hline Fi-Wi & Fiber-wireless \\
Wi-Fi & Wireless fidelity \\
P2P & Peer-to-peer \\
PON & Passive optical networks \\
EPON & Ethernet passive optical network \\
GPON & Gigabit passive optical network \\
OLT & Optical line terminal \\
ONU & Optical network units \\
LP & Linear programming \\
RN & Remote node \\
WMN & Wireless mesh network \\
RoF & Radio-over-fiber \\
R\&F & Radio and fiber \\
RAU & Remote antenna unit \\
\hline
\end{tabular}

nearest ONU through the closest wireless mesh router, then sent to OLT, and afterwards the traffic is sent to Internet from OLT. For example, a mesh client $a$ would like to communicate with mesh client $b$, traffic will be routed from mesh client $a$ to its nearest ONU through wireless mesh router, then to the OLT from the nearest ONU through RN, then the OLT broadcasts the traffic back to ONUs, and the ONU which is the closest to mesh client $b$ can send the traffic to client $b$ through the nearest wireless mesh router. Figure 1 shows a typical Fi-Wi network architecture that allows such P2P communications.

2.2. An Example Model of Fi-Wi. The architecture of Fi-Wi network is designed as a directed graph $G=(N, E)$, where $N$ is the combination of the OLT denoted as $u_{0}$, a set of ONUs (gateways) denoted as $N_{G}$, and a set of wireless mesh routers denoted as $N_{M}$.

The single-channel wireless routers in the wireless subnetwork of Fi-Wi networks are used with single channel with channel capacity $C$. All wireless nodes work on the same fixed transmission power with the transmission range $R_{T}$ and the interference range $R_{I}$. Generally, $R_{I}$ is $\beta$ times of $R_{T}$, where $\beta>1$. There are wireless links between any two wireless nodes if they are within the transmission range of each other. For any two nodes, $u \in N_{M} \cup N_{G}$ and $u \in N_{M}$, there are wireless links $e_{u v}, e_{v u} \in E$ if they are in the transmission range of each other.

In Fi-Wi network, ONUs can communicate with each other by sending the traffic to the OLT, and then OLT sends the traffic back to all ONUs. In any two ONU nodes, $u, v \in N_{G}$, the direct communication $e_{u v}\left(e_{v u} \in E\right)$ with infinite capacity (see Figure 2) can be placed because the bandwidth capacity provided by the PON subnetwork is much higher than the capacity provided in the wireless subnetwork. If this communication happens in the PON subnetwork, there will be no wireless interference, and the interference set of such links is set to be $\emptyset$. If two gateways in traditional WMNs are within transmission range of each 


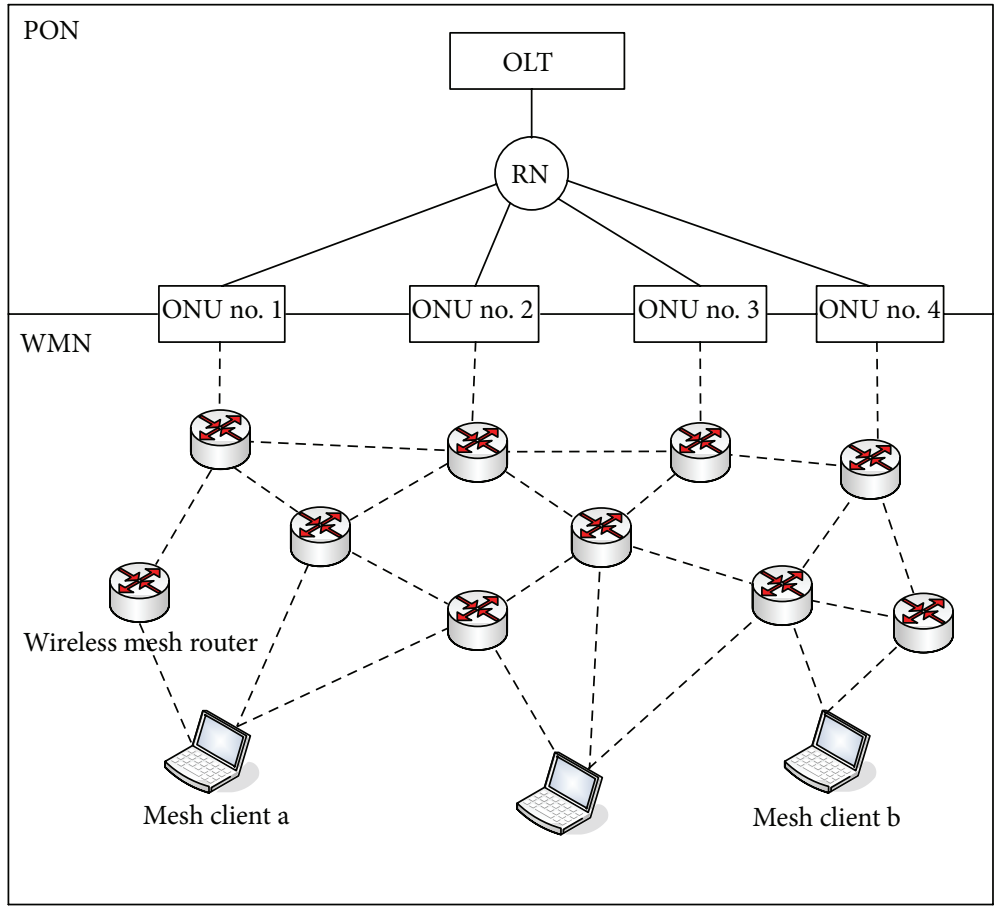

FIgURE 1: Typical architecture of a Fi-Wi Network.

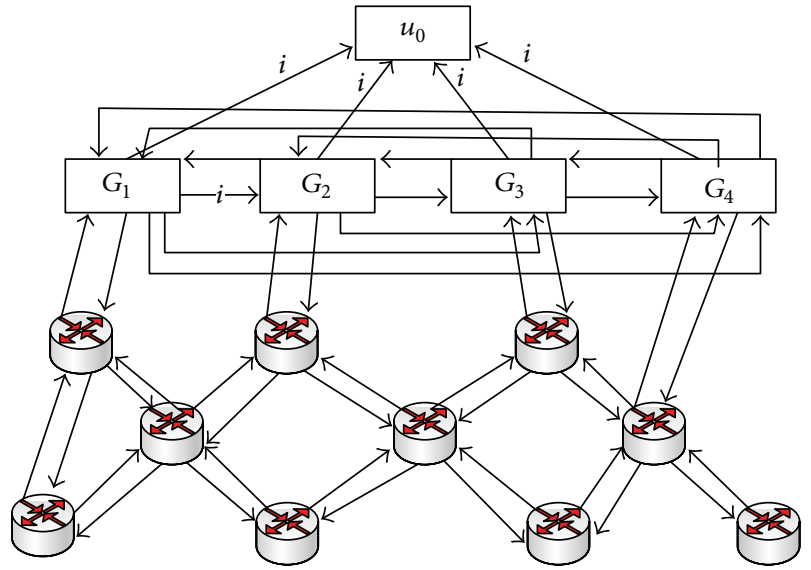

FIGURE 2: Architecture of Fi-Wi Network with multiple ONUs, where all ONUs (i.e., $N_{G}$ ) are connected with each other with the wired link of infinite capacity $(i)$ and also connected to OLT $\left(u_{0}\right)$ with the wired link of infinite capacity $(i)$.

other, they can communicate with each other through wireless transmissions. If they are not covering each other with their transmission ranges, there is no direct communication between them. There may be other types of architectures as well. In the work presented in [5], a unique node representing the wired network is introduced alongside a unidirectional link from each gateway to the unique node with infinite capacity. A unidirectional link with infinite capacity can be placed for the communication from each ONU to the OLT. This communication has no wireless interference, and the interference set of such types of links is set to be $\emptyset$.
For the interference model, we can apply the same model as is presented in the works like $[1,6]$ as $e_{u v} \in \operatorname{Inter}\left(e_{u v}\right)$ because the set of wireless links that interfere with $e_{u v}$ is denoted by $\operatorname{Inter}\left(e_{u v}\right) \subseteq E$. Later in this paper, we will use these notations and sample architecture to describe the network traffic and routing issues.

2.3. RoF versus R\&F Network Technologies. As basic underlying technologies of Fi-Wi network, both RoF (radio-overfiber) and R\&F (radio and fiber) technologies can be found. Here, we will know in brief how these technologies operate.

2.3.1. RoF (Radio-over-Fiber). In RoF Wi-Fi networks, radiofrequencies (RFs) are carried over optical fiber links between a central office $(\mathrm{CO})$ and multiple low-cost remote antenna units (RAUs) to support variety of wireless applications. RoF networks provide transparency against modulation techniques and are able to support various digital formats and wireless standards in a cost-effective manner. These networks use optical fiber as an analogue transmission medium between a central control office and one or more RAUs (radio antenna units). The CO is in charge of controlling access to both optical and wireless media. However, using an optical distribution system in wireless networks may have a negative impact on the performance of medium access control (MAC) protocols. The additional propagation delay may exceed certain timeouts of wireless MAC protocols, and the network performance would become worse. Figure 3 shows a sample RoF Wi-Fi access network architecture that can be used within a Fi-Wi network. 


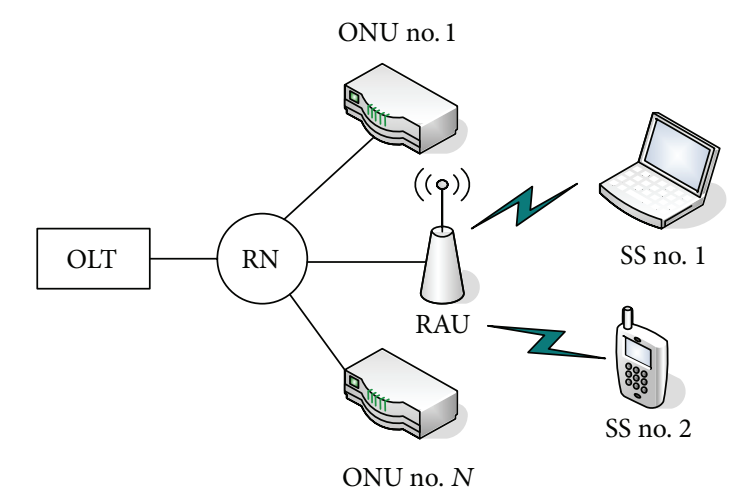

RAU: Remote antenna unit

SS: Subscriber station

FIgURE 3: RoF Wi-Fi access network architecture: PON and its wireless extension RAU.

2.3.2. R\&F (Radio and Fiber). R\&F Wi-Fi networks use two different MAC protocols separately for the optical and wireless media to access PON and WMN. Protocol translation must be done at the interface of optical and wireless segments by an appropriate optical-wireless device, such as optical network unit-base station (ONU-BS). In this case, wireless MAC frames do not have to travel along the optical fiber to be processed at the central control office but simply have to traverse their associated access point and remain in the wireless mesh network. In WLAN-based (wireless local area network-based) R\&F networks, access control is done locally inside the wireless subnetwork without involving any central control office so that it can avoid the negative impact of fiber propagation delay on the network throughput and it can also reduce the interferences in wireless subnetwork. R\&F networks are the most suited architecture to build WLANbased Fi-Wi networks of extended coverage. Figure 4 shows a sample R\&F Wi-Fi access network model that can be applied in a Fi-Wi network infrastructure.

\section{A Closer Look at the Works on Fi-Wi}

In the last few years, Fi-Wi network structures have been used extensively in many areas, and some researchers have been focusing on architectural issues like finding the optimal ONU placement to maximize the network throughput, reconfiguration of the PON structures, and routing algorithms in the wireless subnetwork of these networks. The strategies of the network throughput and the gateway deployment in wireless mesh subnetwork play significant roles. Among various published works on these aspects, we will discuss some of the works in this section to have a better understanding of the topic.

Reference [7] presents an idea on how to protect FiWi network structure from multiple segment failures. The authors term their schemen as RPMF (ring-based protection considering multiple failures). In this scheme, a backup ONU is designated in each segment of the network and all the segments are clustered in the network. Then, in each

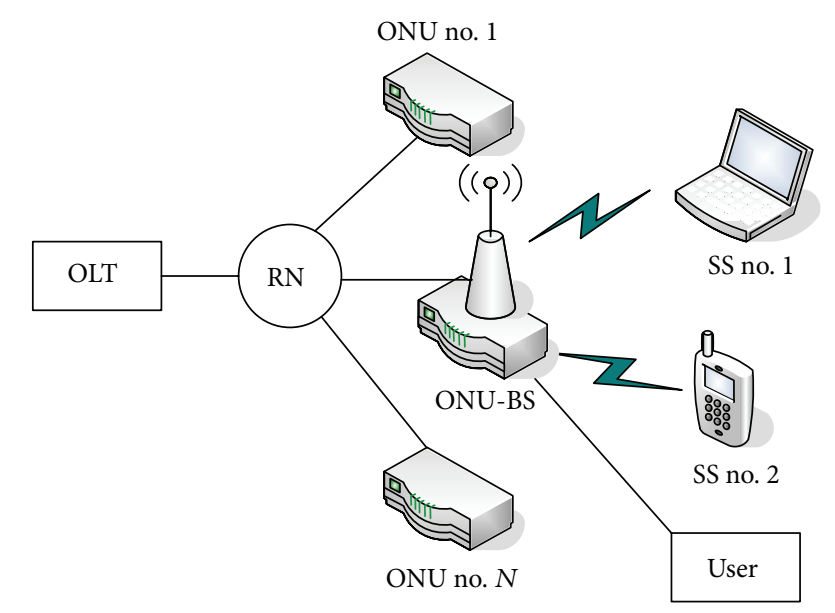

ONU-BS: Optical wireless unit-base station

SS: Subscriber station

FIgURE 4: R\&F Wi-Fi access network architecture: PON and its wireless extension ONU-BS.

cluster, the authors deploy backup fibers among the backup ONUs in different segments to build a protection ring. This scheme guarantees that the segments in each cluster have enough residual capacity to carry the interrupted traffic in the worst failure scenario such that the network can tolerate the simultaneous failures of multiple segments. If a segment fails, the scheme allows the failed segment to transfer its traffic into any other available segments in the same cluster with two alternative paths along the protection ring. Thus, this scheme can tolerate the simultaneous failures of any one segment and its backup fiber.

An integrated flow assignment and fast resequencing algorithm are suggested in [6] to reduce the out-of-order probability of packets departing from the OLT with virtually zero resequencing delay. Mixed integer programming (MIP) Model [8] and simulated annealing (SA) algorithm [9] are designed to minimize the average distance of any wireless mesh router to its neighbor ONU. In order to improve bandwidth utilization, a dynamic reconfiguration algorithm in WDM (wavelength-division multiplexing) PONs is proposed in the work [10]. Delay-aware routing algorithm (DARA) [11] and capacity and delay aware routing algorithm (CaDAR) [12] have been suggested to highlight the issue of network routing in the Fi-Wi network. Moreover, the study in [13] proposed a centralized integrated routing to accomplish the load balancing at ONUs and to maximize the network throughput.

Reference [14] proposes a new scheduling algorithm called modified deficit round robin scheduling (MDRR) to increase the throughput of TCP and to ensure possible inorder packet delivery at the destination. MDRR depends on the DRR (Round Robin Scheduling) fundamentals. In MDRR, the quantum value given to the queues is based on the weight associated with them. It provides better quality of service and eventually leads to changing the throughput, latency, jitter, and packet overflow at the queues. 
The work presented in [15] shows that in a wireless network with $n$ nodes, the per-node throughput is $\theta(1 / \sqrt{n \log n})$ using the random node placement and communication mode. It becomes $\theta(1 / \sqrt{n})$ using the optimal node placement and communication pattern. The joint channel assignment and routing algorithms are proposed $[5,16]$ in multiradio, multichannel WMNs to maximize the network throughput under certain fairness constraints. The work in [17] tries to attain the fairness guarantee among different traffic flows and to maximize the network throughput. Some works like [1823] address signal processing issues for better performance of the links in Fi-Wi structure.

The gateway deployment scheme of wireless networks has been studied as well. The analysis in [24] focuses on the downstream controlling of Fi-Wi networks and proposes a traffic distribution scheme which utilizes an aspect of EPON to properly distribute traffic load among ONUs/GWs (gateways). The authors adopt a hop count limitation mechanism to avoid throughput degradation caused by increased wireless interference and effects of cross traffic in the WMN. The results show a trade-off relationship between fair load balancing among ONUs/GWs (gateways) and high throughput for end users.

Moreover, a way to deploy the minimal number of gateways in WMNs is pointed out in $[25,26]$ so that the QoS (Quality of Service) requirements are fulfilled. Algorithms for gateway placement under different wireless models have been proposed in [27], and the algorithm is also accounted for the traffic load variance from different users. Reference [28] studies WMN's optimal model to minimize the cost of overall network installation and to provide full coverage to all wireless clients. Reference [29] presents how to add new gateways into existed WMNs to achieve more network capacity. However, these works are only focused on the traffic to the Internet, not for peer-to-peer communications.

Reference [30] points out the impact brought by the wireless-optical-wireless mode on the network throughput in Fi-Wi networks under certain ONU placement. In [30], the performance improvements of peer-to-peer communications between two mesh routers in the same Fi-Wi network have been investigated, and the use of optical lines in PONs (instead of numerous wireless multihop connections in WMN for the peer-to-peer communication between distantly positioned source and destination mesh routers) has been proposed. According to [30], utilizing PONs for communication between source and destination mesh routers mitigates the throughput reduction and end-to-end delay, caused by wireless interference (which is increased by many hops involved in a $\mathrm{WMN}$ ).

After going through all these works mentioned above, we have found that only a few works talk about Fi-Wi network architectures that provide alternative routing for peer-to-peer communications, which can utilize the high bandwidth provided by PON subnetwork and could ease interferences in wireless subnetwork to increase the network throughput. In the next section, we will look into the case of throughput enhancement in such network as this is the main facility for which Fi-Wi networks are considered to be appealing.

\section{Quality-of-Service (QoS) Issues}

Quality-of-service (QoS) plays a key role in running various multimedia applications and services over Fi-Wi networks because QoS techniques are used to measure bandwidth, to detect changing network conditions such as congestion or availability of bandwidth, and to prioritize network traffic. In fact, better QoS of Fi-Wi is the main attractive point in choosing such kind of network for the implementation over other alternatives. QoS technologies can be applied to prioritize traffic for latency-sensitive applications such as voice or video and to control the impact of latency-insensitive traffic. However, the variability of wireless channel conditions and mobility of wireless end users might change the quality of service, which requires quick response time.

Recently, various QoS techniques were developed for FiWi networks. QoS support is achievable by managing the network layer. Different techniques might be used for the design of hybrid Fi-Wi networks to deliver QoS-enabled services for end-users. In [31], centralized and distributed scheduling approaches were examined for the integration of EPON and WiMAX. The simulation results show an improved network throughput and end-to-end delay for different QoS demands. The integrated QoS-aware dynamic bandwidth allocation (DBA) scheme proposed in [32] supports bandwidth fairness at the ONU-BS (base station) interface which sends optical bandwidth requests to the optical line terminal (OLT) based on the status of its queues. The reported results show improved network throughput, delay, and bandwidth utilization.

To gain maximum network throughput in both wired and wireless network traffic, key QoS metrics, such as packet loss, bandwidth, delay, jitter, and packet-error ratio, are important. The design of suitable routing algorithms is another mean to improve QoS support in Fi-Wi networks.

\section{TCP Performance in Fi-Wi Network}

Between node pairs of Fi-Wi network, transmission control protocol (TCP) provides reliable data transfer. In order to prevent network congestion and improve throughput, packets of a flow are sent through multiple paths in wireless subnetwork and then sent to the OLT through ONUs in the optical subnetwork. OLT has responsibility to transmit the packets to their destinations. As all traffic in such network is sent to OLT, OLT works as a convergence node, which can resequence the packets before those are sent to the Internet or to another wireless client inside wireless subnetwork.

When OLT sends the packets to their destination, packets may arrive out of order because of the variant delay along multiple paths in Fi-Wi network. When this happens, a duplicate acknowledgment (ACK) is sent by TCP receivers for each out-of-order (OOD) segment. When TCP sender receives three duplicate ACKs, sender would assume that a segment has been lost and the network is congested. The lost packet can be recovered by retransmitting the lost packet from TCP senders when a duplicate ACK is received. The congestion avoidance is performed after the fast retransmission and fast recovery. 
However, there is a problem when many packets arrive dynamically from different flows with packet reordering at OLT, where the OLT maintains a queue for each flow and the processing is divided into multiple equal time slots. Each divided time slot can send out only one packet from the OLT to the Internet or another wireless client inside wireless subnetwork.

The OLT needs to decide which flow's packets should be sent and which packets from selected flow should be sent to their destinations. This will activate the fast retransmission and fast recovery because of the duplicate ACKs and then will cause additive increase in TCP's congestion window size (cwnd) in the network where reordered packets are widespread. This action can affect the TCP performance in Fi-Wi network.

In order to increase the throughput of TCP and to reduce the out-of-order packets from OLT, a suitable packet scheduling algorithm is needed. Scheduling is done for load balancing and achieving QoS. In Fi-Wi network, the scheduling algorithm is used at OLT to resequence the packets of each flow to provide in-order arrivals at the destination. The resequencing can also be done at the end system. The resequencing at OLT must be done fast so that too much delay would not be introduced to the resequenced packets. The most challenging part of making scheduling decision is to maintain the number of duplicate ACKs for each queue. There are some fundamental scheduling algorithms which can be used in packet resequencing such as FIFO (first-infirst-out), DRR (deficit round robin), and MDRR (modified deficit round robin scheduling).

OLT maintains a temporal priority queue system (TPQ) with two priority levels for each flow. The out-of-order packets to the OLT are put to the high priority level and otherwise to the low priority level; both levels are served in the first-in-first-out (FIFO) order. In FIFO, the oldest process in the ready queue is selected for running when the current running process is executed. The average waiting time is quite long in FIFO scheduling algorithm. Deficit round robin (DRR) can handle packets of variable size without knowing their mean size. A maximum packet size number is subtracted from the packet length, and packets that exceed that number are held back until the next visit of the scheduler. Modified deficit round robin scheduling (MDRR) algorithm depends on the DRR fundamentals. In MDRR, the quantum value given to the queues is based on the weight associated with them.

According to the work in [14], MDRR scheduling algorithm is more effective than FIFO and DRR to provide inorder arrivals at the destination. The results show MDRR is more efficient in assuring fairness among the different flows and reducing the effect of packet reordering and the required buffer size in the OLT.

\section{Traffic Routing Issues in Fi-Wi}

The integration of the optical networks and wireless networks offers an attractive and feasible solution to measure the traffic flow and count the number of hops in Fi-Wi network. Although there are many routing algorithms to derive maximum network throughput, linear-programming(LP-) based routing algorithm $[5,33]$ is more suitable because there are multiple ONUs and fiber wired communications between ONUs. Therefore, LP-based routing algorithm is introduced in this section for future study. It should be noted that LP-based routing technique (which is optimal routing scheme) is not likely to be used in the practical scenarios as theory often does not directly translate to the practical implementation cases. Therefore, a practical routing scheme can be applied for traffic between wireless peer in Fi-Wi network to achieve more network throughput gain from the wireless-optical-wireless mode. In this section, Fi-Wi network traffic characteristics and various routing models are discussed, which are adopted from $[5,30,33]$.

6.1. Multiflow Traffic Demand. Multipath routing is utilized in order to increase the total network utilization, higher aggregate bandwidth, less delay, and better load balancing which improve the network throughput and support upstream, downstream, and peer-to-peer communication efficiently.

In the network architecture shown in Figure 2 (see Section 2.2), there are two traffic flows that have to be considered. The first one is the traffic from wireless mesh client to the Internet, and the second one is peer-to-peer communication in Fi-Wi networks. So, let $F$ be the traffic flow vector in the network. Therefore, the flow $f$ in the vector $F$ consists of $s_{f}, d_{f}$, and $r_{f}$ which are the source node, the destination node, and the traffic demand of the flow $f$, respectively. In particular, $s_{f} \in N_{M}$ and $d_{f}=u_{0}$ or $d_{f} \in N_{M}$.

If $d_{f}=u_{0}$, the traffic is for the route to Internet. There are $Q$ traffic flows in $F$. So, let $F_{\text {Inet }}$ be the set of flows routing to the Internet, where $F_{\text {Inet }}=\left\{f d_{f}=u_{0}\right\}$, and let $F_{\mathrm{P} 2 \mathrm{P}}$ be the set of flows for peer-to-peer communication in the network where $F_{\mathrm{P} 2 \mathrm{P}}=\left\{f \mid d_{f} \in N_{M}\right\}$. Then, $F=F_{\text {Inet }} \cup F_{\mathrm{P} 2 \mathrm{P}}$. Each wireless node has traffic to the Internet and traffic to every other wireless node.

6.2. LP-Based Routing Algorithm. As mention in overall architecture, each wireless node has only one radio working on one channel. Therefore, there is no channel assignment issue. To gain maximum network throughput, only we need to consider routing and link scheduling approaches to gain maximum network throughput in peer-to-peer communication, the same LP-based routing algorithm can be used that is proposed in [30]. Let us know about the operating principles of the algorithm.

Let $X_{f}$ where $0 \leq X_{f} \leq 1$ be the percentage of traffic demand routed to the destination node for flow $f$ in the vector $F$. Therefore, $X_{f} r_{f}$ is the traffic routed to the destination node for flow $f$, and $x_{u v}^{f}$ can be assigned as the traffic load of flow $f$ on the link $e_{u v}$ and $x_{u v}$ as the traffic load 
on link $e_{u v}$. Then, we have $x_{u v}=\sum_{i=1}^{\mathrm{Q}} x_{u v}^{f}$. To maximize the network throughput in the Fi-Wi network:

$$
\begin{array}{ll}
\max \quad & \sum_{f=1}^{Q} X_{f} r_{f}, \\
\text { Subject to } & \sum_{v: e_{u v} \in E} x_{u v}^{f}=X_{f} r_{f}, \quad f=1 \cdots Q, u=s_{f}, \\
& \sum_{v: e_{u v} \in E} x_{u v}^{f}-\sum_{v: e_{v u} \in E} x_{v u}^{f}=0, \\
f=1 \cdots Q, \quad \forall u \neq s_{f}, d_{f}, & \\
& \sum_{v: e_{v u} \in E} x_{v u}^{f}=X_{f} r_{f}, \quad f=1 \cdots Q, u=d_{f}, \\
\sum_{u_{u^{\prime} v^{\prime}}: e_{u^{\prime} v^{\prime}} \in \operatorname{Inter}\left(e_{u v}\right)} \sum_{f=1}^{Q} x_{u^{\prime} v^{\prime}}^{f} \leq c, \quad \forall e_{u v} \in E, \\
x_{u v}^{f} \geq 0, \quad f=1 \cdots Q, \quad \forall e_{u v} \in E, \\
0 \leq X_{f} \leq 1, \quad f=1 \cdots Q .
\end{array}
$$

The flow conservation at each node in the network is ensured by (2), (3), and (4). Equation (5) shows the wireless link interference constraint in the network. Equations (6) and (7) make sure that both $x_{u v}^{f}$ and $X_{f}$ are with nonnegative values.

6.3. Practical Routing Scheme. Let us consider the scenario presented in Figure 5. To practically use any routing scheme for the peer-to-peer communication, the traffic routing has two choices between client $a$ and client $b$.

Choice 1. The first choice is to pass traffic through the available shortest path between clients within the wireless subnetwork.

Choice 2. The second choice is to send traffic through the wireless-optical-wireless mode.

From these two choices, it is better to choose the traffic routing with smaller hop counts because the wireless network throughput will decrease if the hop count increases. If destination client is reached before the ONU, traffic is directly routed to destination client from source client. In this case, routing of the traffic would be the same as traditional wireless mesh network.

In the Fi-Wi network and traditional WMNs, the traffic from wireless clients to the Internet will always be routed to the nearest $\mathrm{ONU}$ of the client through the wireless shortest path, and the peer-to-peer communication within the wireless network in WMN will be through the shortest path between the peer clients. This is what is called the practical routing scheme for Fi-Wi networks.

As the PON subnetwork would be fast enough considering the two parts involved in Fi-Wi structure, our main concern would be finding shortest path in the wireless segment. To find out the shortest path in wireless mesh network, a scheme like that is presented in [34] could be used. In this work, the authors use $k$-shortest path approach which computes several $(k)$ candidate paths in each node towards source and then chooses the best (shortest one) among them. Each node maintains a set of $k$-shortest paths toward the source. The routing phases are route discovery, route reply, and route maintenance. The admission control guarantees that the flow on each link is no less than the required bandwidth. Further investigation can be done on this issue, and other shortest path routing mechanisms may be devised.

\section{Challenges and Future Prospects of Research}

In this section, we will present the issues on which more investigations could be performed in the coming days. Though many solutions may be available on different aspects of the network, some issues are yet to find any standardized or optimal solution. In fact, often the challenges remain with the application scenario and requirements of a particular network setting. Let us find out more on these.

7.1. Performance. The performance of Fi-Wi network relies on the design of gateway nodes (ONUs) which is very important in Fi-Wi integration. The bandwidth allocation, routing, and link scheduling are also important functions in the network design. Link scheduling is used in wireless mesh networks to guarantee interference-free transmission on the shared wireless medium. Performance of such network's uplink could also degrade significantly because of the multipath dispersion introduced by the wireless link and the nonlinear distortion caused by the radio-over-fiber (RoF) link [35]. To illustrate a bit, in optical fibers, multipath dispersion occurs in a wide core because light travelling along the axis of the core travels a shorter distance per meter of a fiber than light that repeatedly undergoes total internal reflection. A pulse of light sent along a wide core would become longer than that it has to be. If it is too long, it would merge with the next pulse. This creates multipath distortion. On the other hand, nonlinear distortion here means the phenomenon of a nonlinear relationship between the "input" and "output" signals of the electronic device (which is sending signals). These issues may not be measured from the receiver's side; hence, channel equalization is a challenging task in Fi-Wi network. Channel equalization means the process of adjusting the balance between frequency components within an electronic signal for the network's smooth operation and better performance. There are some works like $[35,36]$ to improve performance addressing this issue; however, devising an optimal solution applicable in various scenarios is always an open challenge.

7.2. Routing. Routing, like any other network, is a very important issue in Fi-Wi network since multihopping has a great impact on network performance degradation in WMN. Routing algorithms will allow wireless traffic to use the capacity offered by optical gateways. Routing algorithms 


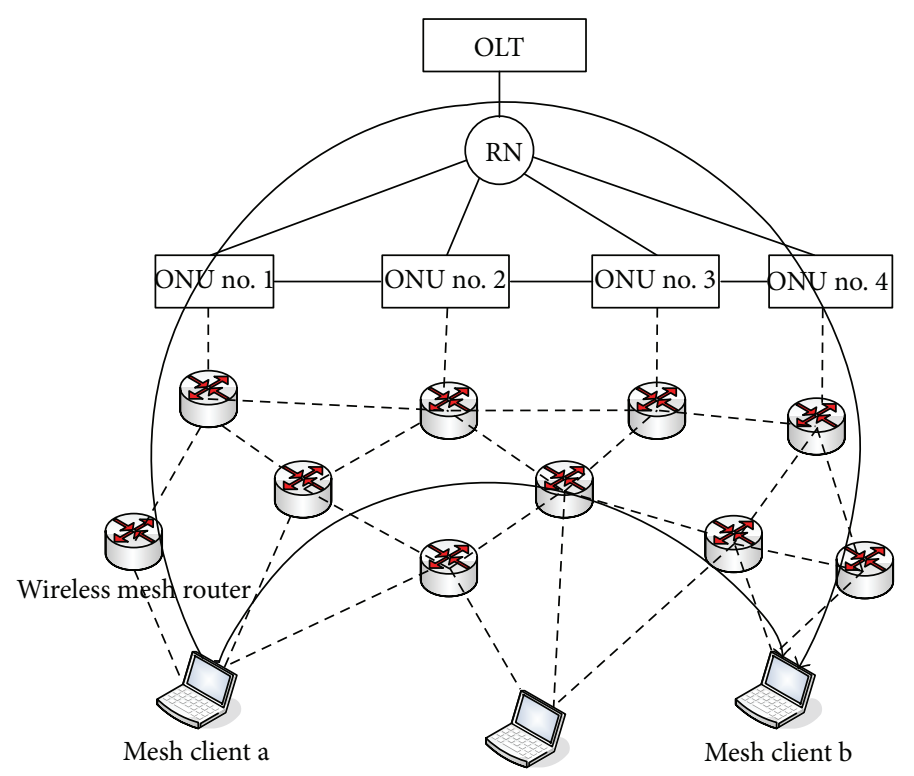

FIGURE 5: Architecture of Fi-Wi network with practical routing scheme.

provide layer-3 functionality and several efficient algorithms which help reduce the number of hops in a WMN. However, these algorithms cannot support the functionalities that take place at a lower level. Intensive research works on upstream scheduling algorithms for PON or related system have been contributed in some previous papers [37-40]. In the FiWi network, traffic flows arrive at the gateway node from clients via wireless upstream links which can lead to signal attenuation, fading, interference, and noise. Therefore, most of the existing algorithms cannot be directly adapted without taking features of wireless links into consideration.

7.3. Architectural Issues. Many researchers have proposed several integrated network architectures which combine optical and wireless communication to gain maximum network throughput. Some of the basic architectures [41] are independent architecture and radio-over-fiber (RoF). In the independent architecture, the ONU is directly connected with base station (BS) via a common standardized interface. There is no direct wireless link between the ONU and the clients. The main advantage of this architecture is the independent deployment of the two access networks. However, the communication between ONU and BS, which exchanges information, is unavoidable because two domains are separate. Therefore, control signaling messages may suffer a delay and cause traffic overhead. Reducing various types of delays in both parts of Fi-Wi is always an open issue when services like video-on-demand (VoD), live streaming, video conferencing, and other multimedia delivery are considered $[42,43]$.

In radio-over-fiber $(\mathrm{RoF})$, its architecture consists of a central control office (CO) and a remote antenna unit connected by an optical fiber link on which the microwave signals are distributed. The advantages of RoF architecture is low attenuation loss, immunity to radio frequency interference, and reduced power consumption. However, for analog modulation and analog signal transmission, the signal impairments such as noise and distortion become challenging issues to handle.

7.4. Resource Utilization. Each technology in Fi-Wi uses its own MAC protocol for serving client traffic. If these technologies work independently, the large amount of available resources will not be utilized in optical networks. Therefore, the use of hybrid integrated MAC protocol is very important in order to efficiently utilize the available resources and achieve high overall network throughput. These protocols must support end-to-end QoS so that Service Level Agreements (SLAs) could be satisfied and clients would receive guaranteed service.

7.5. Supporting Different Technologies. An important feature is the network's capability to support peer-to-peer communication and multicasting technologies. File sharing and video conferencing in peer-to-peer communication are examples of where users exchange large amounts of data. This kind of traffic can be served more efficiently by using decentralized resource allocation mechanisms to avoid possible network bottlenecks. The same content must be delivered to multiple users in services like video on demand (VoD) and Internet Protocol TeleVision (IPTV). Multicasting supports one unique flow which is simultaneously transmitted to many users avoiding wastage of resources. These are very important features of current and future networks and must be explored in detail for Fi-Wi networks.

7.6. Network Throughput. Concerned with the traffic to the Internet, each wireless router should be able to reach at least one ONU for the Internet access. Each wireless node would calculate the shortest path with minimal hop count 
to ONUs and select one ONU as a primary gateway for Internet access. The network throughput would be high when the traffic is only directed for the Internet access. However, the network throughput would decrease when the traffic demand increases in peer-to-peer communication, because the interferences would increase in the wireless network. Although the network throughput can be possibly less when node degrees are increased in the wireless network, we can increase the network throughput in Fi-Wi network and in the traditional WMN by applying more traffic demand for each flow of the network.

7.7. Energy Consumption. A very important issue of the FiWi network implementation is its energy consumption [44]. Though many parts could be supported by constant power supply sources, preserving energy or energy cost is a critical issue to make it more suitable and attractive over other available technologies. As we are moving towards the green information technology (IT) or green computing era, it is very important to know and study the energy consumption of every device in the Fi-Wi network. With the advancements of associated networking technologies, it is expected that the FiWi network would be more widely used for various consumer electronics applications in the coming days.

7.8. Green Computing. As the notion of green computing [45, 46] is thriving today, like any other networking technologies, Fi-Wi needs to address the environmental issues as well. Involvement of large number of fibers and associated devices to support the infrastructure would produce heat and green house gas which need to be addressed if Fi-Wi networks are to replace the relatively slower old or currently established network backbones. Hence, reducing green house gas emission or heat (thus not contributing to global warming) would be an integral part of future research in Fi-Wi areas as well.

7.9. Integration with Smart Grid. A very exciting area of further investigation could be combining Fi-Wi network with smart grid [47]. For faster operation of smart grid, the Fi-Wi could work as a backbone. Another interesting area could be a cloud infrastructure with Fi-Wi support. As reduction of time in many commercial and business computing applications is a major issue, Fi-Wi's fast data exchanging infrastructure may be able to facilitate such Fi-Wi Cloud!

\section{Conclusion}

To summarize the overall contribution of this paper, the architecture of Fi-Wi network is investigated based on previously conducted research works. Various aspects of this network have been analyzed to gain some insights of the technology. We have investigated the network formation issues, performance issues, possible routing mechanisms, and past works on separate underlying issues. Our contribution is that we have identified the areas for conducting further studies and the possibility of merging Fi-Wi technology in the mainstream networking research. In general, Fi-Wi networks form a powerful platform for the support and creation of long distance, flexible, and high-bandwidth communications as well as for the future unforeseen applications and services. Therefore, though many issues are mentioned in our work, many other related issues to this network architecture are needed to be explored in future with more details.

\section{References}

[1] G. R. Hiertz, D. Denteneer, S. Max et al., "IEEE 802.11s: the WLAN mesh standard," IEEE Wireless Communications, vol. 17, no. 1, pp. 104-111, 2010.

[2] N. Ghazisaidi, M. Scheutzow, and M. Maier, "Survivability analysis of next-generation passive optical networks and fiberwireless access networks," IEEE Transactions on Reliability, vol. 60, no. 2, pp. 479-492, 2011.

[3] N. Ghazisaidi and M. Maier, "Fiber-wireless (FiWi) access networks: challenges and opportunities," IEEE Network, vol. 25, no. 1, pp. 36-42, 2011.

[4] A. Ngoma, H. Yang, and J. George, "Integrated fiber-wireless networks: what, why and how," in Proceedings of the IEEE Africon, pp. 1320-1325, Livingstone, Zambia, September 2011.

[5] M. Alicherry, R. Bhatia, and L. E. Li, "Joint channel assignment and routing for throughput optimization in multiradio wireless mesh networks," IEEE Journal on Selected Areas in Communications, vol. 24, no. 11, pp. 1960-1971, 2006.

[6] J. Wang, K. Wu, S. Li, and C. Qiao, "Performance modeling and analysis of multi-path routing in integrated fiber-wireless networks," in Proceedings of the IEEE INFOCOM, pp. 1-5, March 2010.

[7] Y. Liu, L. Guo, and B. Gong, "Ring-based protection scheme for survivable fiber-wireless (FiWi) access network considering multiple failures," in Proceedings of the 1st IEEE International Conference on Communications in China (ICCC '12), pp. 285290, August 2012.

[8] S. Sarkar, H.-H. Yen, S. Dixit, and B. Mukherjee, "A mixed integer programming model for optimum placement of base stations and optical network units in a hybrid wireless-optical broadband access network (WOBAN)," in Proceedings of the IEEE Wireless Communications and Networking Conference (WCNC '07), pp. 3907-3911, March 2007.

[9] S. Sarkar, B. Mukherjee, and S. Dixit, "Towards global optimization of multiple ONU placement in hybrid optical-wireless broadband access networks," in Proceedings of the Joint International Conference on Optical Internet and Next Generation Network (COIN-NGNCON '06), pp. 65-67, July 2006.

[10] S. Bhandari and E. K. Park, "Hybrid optical wireless networks," in Proceedings of the International Conference on Networking, International Conference on Systems and International Conference on Mobile Communications and Learning Technologies (ICNICONSMCL '06), p. 113, 2006.

[11] S. Sarkar, H.-H. Yen, S. Dixit, and B. Mukherjee, "DARA: delay-aware routing algorithm in a hybrid Wireless-Optical Broadband Access Network (WOBAN)," in Proceedings of the IEEE International Conference on Communications (ICC'07), pp. 2480-2484, June 2007.

[12] A. Reaz, V. Ramamurthi, S. Sarkar, D. Ghosal, S. Dixit, and B. Mukherjee, "CaDAR: an efficient routing algorithm for a wirelessoptical broadband access network (WOBAN)," Journal of Optical Communications and Networking, vol. 1, no. 5, pp. 392-403, 2009. 
[13] W.-T. Shaw, S.-W. Wong, N. Cheng et al., "Hybrid architecture and integrated routing in a scalable optical-wireless access network," Journal of Lightwave Technology, vol. 25, no. 11, pp. 3443-3451, 2007.

[14] G. Sivakumar and A. V. Ramprasad, "Analysis of FiWi networks to improve TCP performance," in Proceedings of the International Conference on Computing, Communication and Applications (ICCCA '12), pp. 1-6, February 2012.

[15] P. Gupta and P. R. Kumar, "The capacity of wireless networks," IEEE Transactions on Information Theory, vol. 46, no. 2, pp. 388404, 2000.

[16] M. Alicherry, R. Bhatia, and L. Li, "Joint channel assignment and routing for throughput optimization in multi-radio wireless mesh networks," in Proceedings of the 11th Annual International Conference on Mobile Computing and Networking (MobiCom '05), pp. 58-72, September 2005.

[17] R. Draves, J. Padhye, and B. Zill, "Routing in multi-radio, multihop wireless mesh networks," in Proceedings of the 10th Annual International Conference on Mobile Computing and Networking (MobiCom '04), pp. 114-128, October 2004.

[18] Y. Zhao, X. Pang, L. Deng et al., "Experimental demonstration of $5-\mathrm{Gb} / \mathrm{s}$ polarization-multiplexed fiber-wireless MIMO systems," in Proceedings of the International Topical Meeting on Microwave Photonics \& Microwave Photonics Conference (MWP/APMP '11), pp. 13-16, Asia-Pacific, October 2011.

[19] X. Pang, X. Yu, Y. Zhao, L. Deng, D. Zibar, and I. T. Monroy, "Channel measurements for a optical fiber-wireless transmission system in the 75-110 GHz band," in Proceedings of the International Topical Meeting on Microwave Photonics \& Microwave Photonics Conference (MWP/APMP '11), pp. 21-24, Asia-Pacific, October 2011.

[20] L. Deng, M. Beltrán, X. Pang et al., "Fiber wireless transmission of 8. 3-Gb/s/ch QPSK-OFDM signals in 75-110-GHz band," IEEE Photonics Technology Letters, vol. 24, no. 5, pp. 383-385, 2012.

[21] X. Pang, A. Caballero, A. Dogadaev et al., "25 Gbit/s QPSK hybrid fiber-wireless transmission in the $\mathrm{W}$-Band $(75-110 \mathrm{GHz})$ with remote antenna unit for in-building wireless networks," IEEE Photonics Journal, vol. 4, no. 3, pp. 691-698, 2012.

[22] C. Lim, C. Pulikkaseril, and K.-L. Lee, "A study on LCoSbased remote nodes For $60 \mathrm{GHz}$ fiber-wireless links," Journal of Lightwave Technology, vol. 30, no. 19, pp. 3110-3117, 2012.

[23] X. Yu, T. B. Gibbon, R. Rodes, T.-T. Pham, and I. T. Monroy, "System wide implementation of photonically generated impulse radio ultra-wideband for gigabit fiber-wireless access," Jounral of Lightwave Technology, vol. 31, no. 2, pp. 264-275, 2013.

[24] M. Honda, H. Nishiyama, H. Nomura, T. Yada, H. Yamada, and N. Kato, "On the performance of downstream traffic distribution scheme in fiber-wireless networks," in Proceedings of the IEEE Wireless Communications and Networking Conference (WCNC '11), pp. 434-439, March 2011.

[25] B. Aoun, R. Boutaba, Y. Iraqi, and G. Kenward, "Gateway placement optimization in wireless mesh networks with QoS constraints," IEEE Journal on Selected Areas in Communications, vol. 24, no. 11, pp. 2127-2136, 2006.

[26] Y. Drabu and H. Peyravi, "Gateway placement with QoS constraints in wireless mesh networks," in Proceedings of the 7th International Conference on Networking (ICN '08), pp. 46-51, April 2008.
[27] R. Chandra, L. Qiu, K. Jain, and M. Mahdian, "Optimizing the placement of Internet TAPs in wireless neighborhood networks," in Proceedings of the 12th IEEE International Conference on Network Protocols (ICNP '04), pp. 271-282, October 2004.

[28] E. Amaldi, A. Capone, M. Cesana, I. Filippini, and F. Malucelli, "Optimization models and methods for planning wireless mesh networks," Computer Networks, vol. 52, no. 11, pp. 2159-2171, 2008.

[29] J. Robinson, M. Uysal, R. Swaminathan, and E. Knightly, "Adding capacity points to a wireless mesh network using local search," in Proceedings of the 27th IEEE Communications Society Conference on Computer Communications (INFOCOM '08), pp. 1247-1255, April 2008.

[30] Z. Zeyu, W. Jianping, and W. Jin, "A study of network throughput gain in optical-wireless (FiWi) networks subject to peer-topeer communications," in Proceedings of the IEEE International Conference on Communications (ICC '09), pp. 1-6, June 2009.

[31] Y. Luo, S. Yin, T. Wang et al., "QoS-aware scheduling over hybrid optical wireless networks," in Proceedings of the Optical Fiber Communication and the National Fiber Optic Engineers Conference (OFC/NFOEC '07), pp. 1-7, March 2007.

[32] K. Yang, S. Ou, K. Guild, and H.-H. Chen, "Convergence of ethernet PON and IEEE 802.16 broadband access networks and its QoS-aware dynamic bandwidth allocation scheme," IEEE Journal on Selected Areas in Communications, vol. 27, no. 2, pp. 101-116, 2009.

[33] M. Kodialam and T. Nandagopal, "Characterizing the capacity region in multi-radio multi-channel wireless mesh networks," in Proceedings of the 11th Annual International Conference on Mobile Computing and Networking (MobiCom '05), pp. 73-87, September 2005.

[34] Y.-Y. Zeng, B. Xi, Z.-M. Zeng, and H. Wang, "Bandwidth guaranteed shortest path routing in wireless mesh networks," in Proceedings of the 1st International Conference on Communications and Networking in China (ChinaCom '06), pp. 1-3, October 2006.

[35] Y. H. Ng, A. H. Tan, and T. C. Chuah, "Channel identification of concatenated fiber-wireless uplink using ternary signals," IEEE Transactions on Vehicular Technology, vol. 60, no. 7, pp. 32073217, 2011.

[36] C. Lim, A. Nirmalathas, and Y. Yang, "Digitized wireless transport for fiber-wireless systems," in Proceedings of the 13th International Conference on Transparent Optical Networks (ICTON '11), pp. 1-4, June 2011.

[37] K. S. Kim, D. Gutierrez, F. T. An, and L. G. Kazovsky, "Batch scheduling algorithm for SUCCESS WDM-PON," in Proceedings of the IEEE Global Telecommunications Conference (GLOBECOM '04), vol. 3, pp. 1835-1839, December 2004.

[38] W. You, L. Yi, and W. Hu, "Upstream bandwidth allocation supporting differentiated services in OFDMA PONs," in Proceedings of the 17th Opto-Electronics and Communications Conference (OECC '12), pp. 132-133, July 2012.

[39] L. Chen and L. Zheng, "A concentrated-grant-based bandwidth allocation algorithm for ethernet passive optical networks," in Proceedings of the Symposium on Photonics and Optoelectronics (SOPO '12), pp. 1-4, May 2012,.

[40] K. S. Kim, D. Gutierrez, F.-T. An, and L. G. Kazovsky, "Design and performance analysis of scheduling algorithms for WDMPON under SUCCESS-HPON architecture," Journal of Lightwave Technology, vol. 23, no. 11, pp. 3716-3731, 2005. 
[41] Y. Yan, H. Yu, and L. Dittmann, "Wireless channel condition aware scheduling algorithm for hybrid optical/wireless networks," in AccessNets, C. Wang, Ed., vol. 6 of Lecture Notes of the Institute for Computer Sciences, Social Informatics and Telecommunications Engineering, pp. 397-409, 2009.

[42] S. J. H. Zaidi, H. B. Khalil, and A. Bashir, "Reducing delays in FiWi networks," in High Capacity Optical Networks and Enabling Technologies (HONET '11), pp. 133-137, December 2011.

[43] Y. Xu and Y. Li, "ONU patching for efficient VoD service over integrated fiber-wireless (FiWi) access networks," in Proceedings of the 6th International ICST Conference on Communications and Networking in China (CHINACOM '11), pp. 1002-1007, August 2011.

[44] A. Ali, T. Tauqeer, and S. M. H. Zaidi, "Energy aware routing algorithm for FiWi networks (EARF)," in Proceedings of the High Capacity Optical Networks and Enabling Technologies (HONET '11), pp. 89-94, December 2011.

[45] S. Murugesan, "Harnessing green IT: principles and practices," IT Professional, vol. 10, no. 1, pp. 24-33, 2008.

[46] B. Kantarci and H. T. Mouftah, "Ethernet passive optical network-long-term evolution deployment for a green access network," IET Optoelectronics, vol. 6, no. 4, pp. 183-191, 2012.

[47] S. Zeadally, Pathan, A. S. K. Pathan, C. Alcaraz, and M. Badra, "Towards privacy protection in smart grid," Wireless Personal Communications, 2012. 

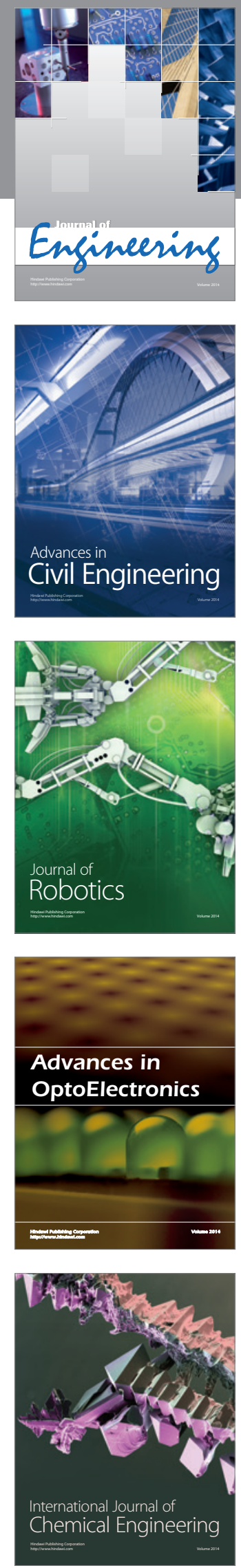

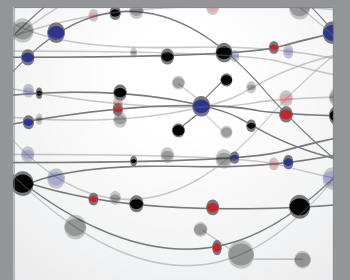

The Scientific World Journal
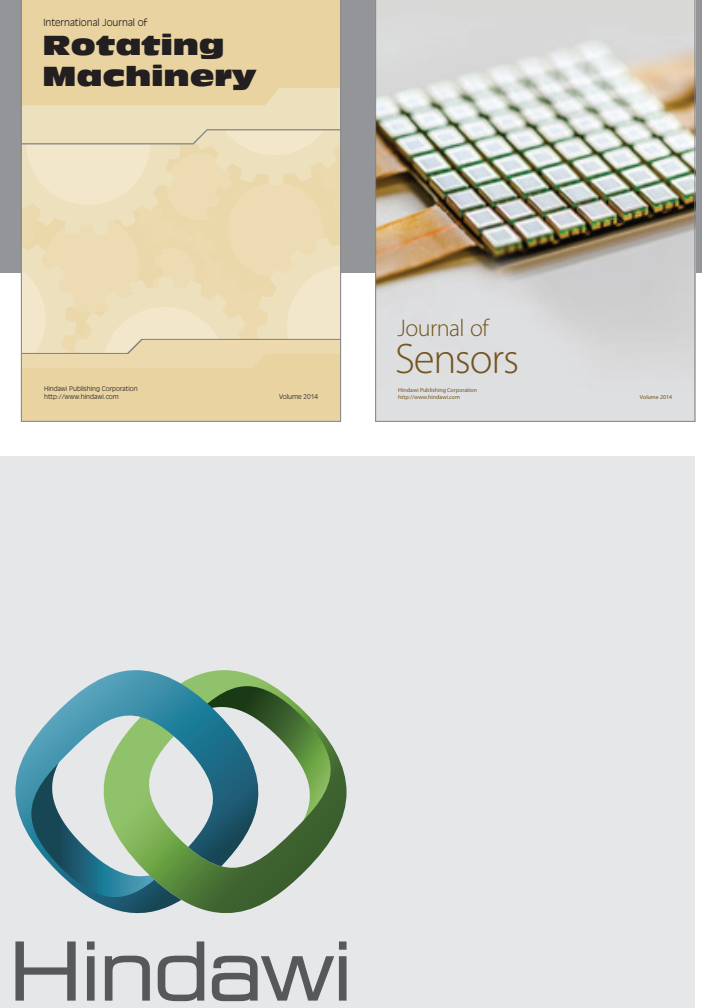

Submit your manuscripts at http://www.hindawi.com
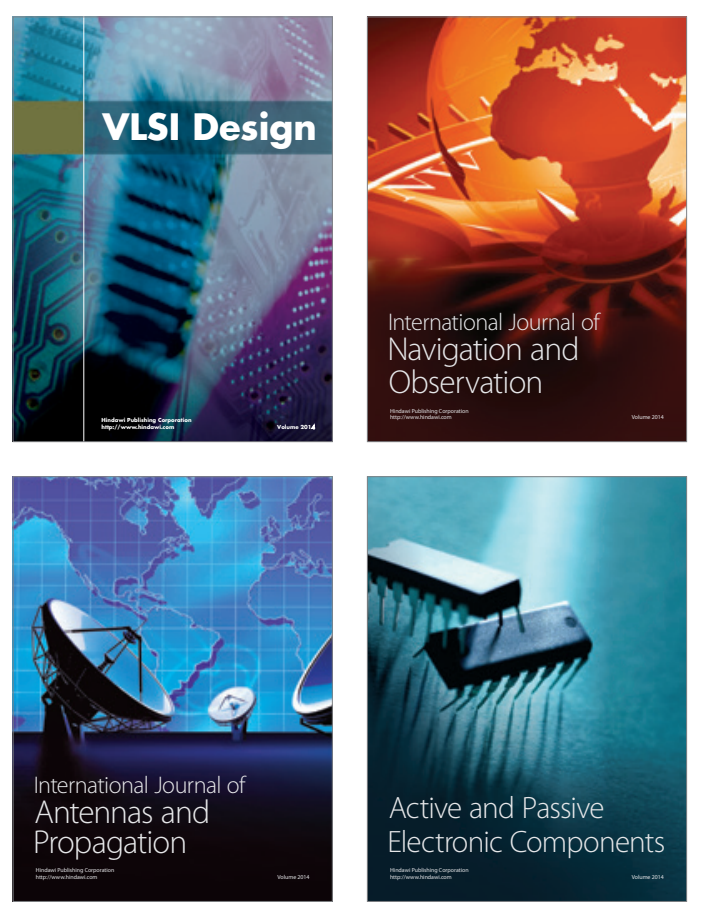
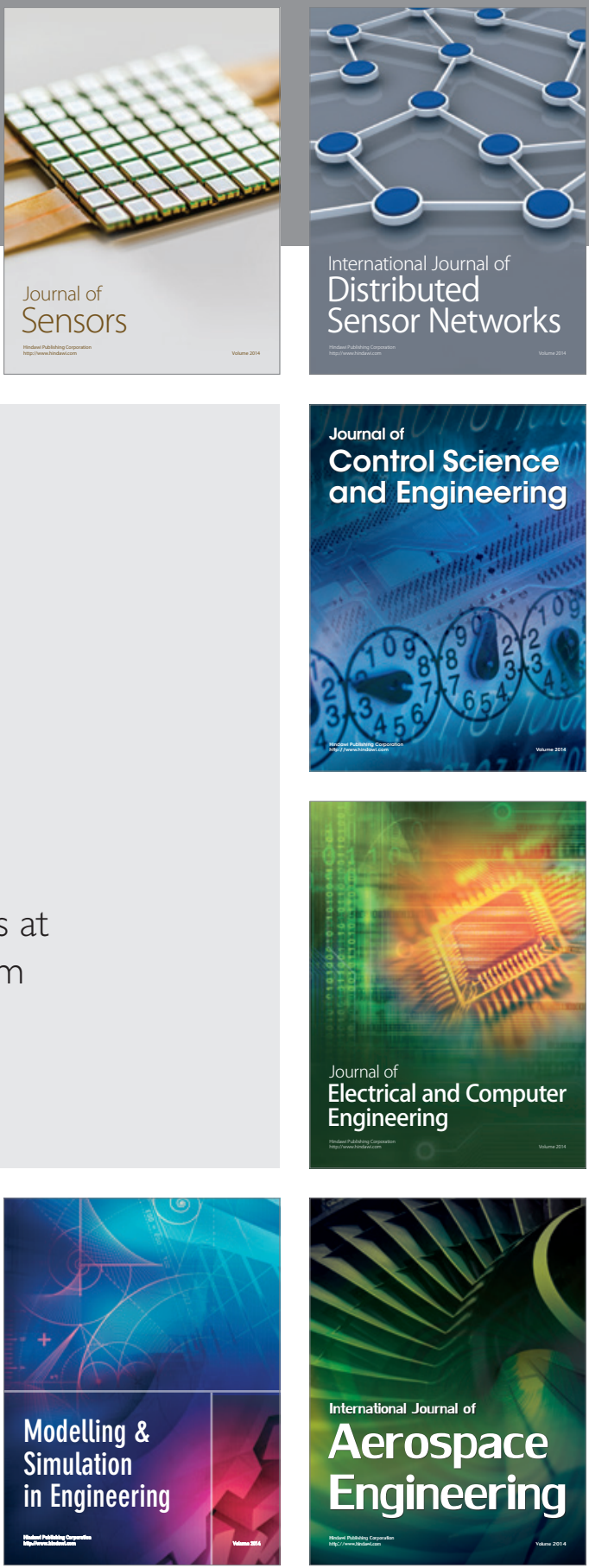

Journal of

Control Science

and Engineering
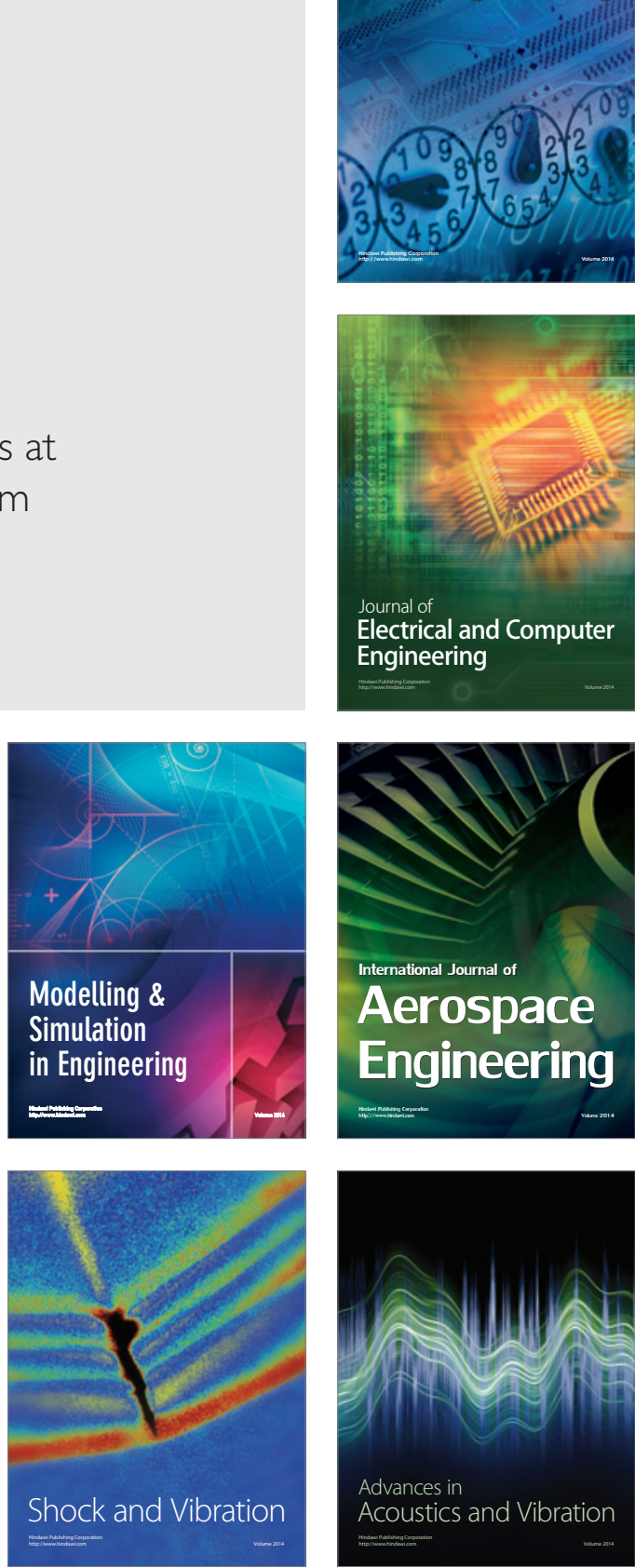